\title{
General and Specific Combining Ability Analysis for Growth and Yield Attributes in Sponge Gourd [Luffa Cylindrica (Roem) L.]
}

\author{
Rajneesh* and V.B. Singh \\ Department of Vegetable Science, ND University of Agriculture and Technology, \\ Kumaranj, Faizabad-224229, India \\ *Corresponding author
}

\begin{tabular}{|c|c|}
\hline & A B S T R A C T \\
\hline Keywords & \multirow{3}{*}{$\begin{array}{l}\text { The present study was conducted with the objective to find out the effects of general and } \\
\text { specific combining ability. The study comprised } \mathrm{F}_{1} \text { hybrids developed through diallel } \\
\text { mating design (excluding reciprocals) with the crosses of } 9 \text { diverse lines of sponge gourd. } \\
\text { The } \mathrm{F}_{1} \text { hybrids and their parents were evaluated for two consecutive years. The } \\
\text { observations were recorded on growth and yield attributes. The Parents, Pusa Chikani }\left(\mathrm{P}_{9}\right) \text {, } \\
\text { NDSG- } 1\left(\mathrm{P}_{1}\right) \text { and NDSG- } 8\left(\mathrm{P}_{8}\right) \text { were found good general combiners for fruit yield along } \\
\text { with number of fruit per plant and some other yield attributes during both the years. } \\
\text { However, lines NDSG- }\left(\mathrm{P}_{1}\right) \text { and Pusa Chikni }\left(\mathrm{P}_{9}\right) \text { were found good general combiners for } \\
\text { maturity traits in both the years. Out of } 36 \text { crosses the best five significant specific crosses } \\
\text { for fruit yield per plant, the crosses } \mathrm{P}_{1} \times \mathrm{P}_{2}, \mathrm{P}_{1} \times \mathrm{P}_{8}, \mathrm{P}_{3} \times \mathrm{P}_{9}, \mathrm{P}_{3} \times \mathrm{P}_{7} \text {, and } \mathrm{P}_{7} \times \mathrm{P}_{8} \text { were } \\
\text { found common over both the years. }\end{array}$} \\
\hline $\mathbf{A} \mathbf{r}$ & \\
\hline & \\
\hline
\end{tabular}

\section{Introduction}

Luffa [Luffa cylindrica (Roem) L. syn. L. aegyptica Mill.] commonly called as sponge gourd, loofah, vegetable sponge or dish cloth. It is one of the most important cucurbit, both as rainy and summer season vegetable which is grown throughout the country and world. It belongs to the family Cucurbitaceae with diploid chromosome number $2 \mathrm{n}=2 \mathrm{x}=26$ which includes about 118 genera and 825 species. It originated in subtropical Asian region particularly India (Kalloo, 1993). Luffa cylindrica (L). and [L. acutangula (L.) Roxb.] are domesticated species. Sponge gourd is an annual and monoecious cucurbit plant. Among vegetables, cucurbits are associated with the origin of agriculture and dawn of human civilization. In food crops, cucurbits are largest producer of biological water and easily digestive and recommended even to sick and frail patients. Its flowers are yellow in colour and showy having five petals. Several workers have emphasized need of parental diversity in optimum magnitude to obtain superior hybrids/superior segregants in the segregating generations (Varalakshmi et al., 1994; Badade et al., 2001 and Islam, 2004). Therefore, efforts should be made to increase the wider use of existing diversity from germplasm collection. 


\section{Materials and Methods}

The experimental materials for the present study comprised of eight promising and diverse inbred lines/parents of sponge gourd selected on the basis of genetic variability from the germplasm stock maintained in the Department of Vegetable Science, N.D. University of Agriculture \& Technology, Kumarganj, Faizabad (U.P.) India. The selected parental lines viz., NDSG-1 $\left(\mathrm{P}_{1}\right)$, NDSG-2 $\quad\left(\mathrm{P}_{2}\right), \quad$ NDSG-3 $\quad\left(\mathrm{P}_{3}\right), \quad$ NDSG-4 $\left(\mathrm{P}_{4}\right)$,NDSG-5 $\left(\mathrm{P}_{5}\right)$, NDSG-6 ( $\left.\mathrm{P}_{6}\right)$, NDSG-7 $\left(\mathrm{P}_{7}\right)$, NDSG-8 $\left(\mathrm{P}_{8}\right)$ and Pusa Chikni $\left(\mathrm{P}_{9}\right)$ (standard check) were crossed in all possible combinations, excluding reciprocals, during summer, 2014 to get $36 \mathrm{~F}_{1}$ seeds for the study on heterosis, 15 fruit yield and yield attributing traits. The experiments were conducted in a Randomized Block Design (RBD) with three replications to assess the performance of $36 \mathrm{~F}_{1}$ hybrids and their 9 parental lines. The crop was planted in rows spaced at 2.5 meters apart with a plant to plant spacing of 0.50 meter. The experiments were sown on $25^{\text {th }}$ February, 2014 and 20 March, 2015. All the recommended agronomic package of practices and protection measures were followed to raise good crops.

\section{Results and Discussion}

General combining ability study helps in making the choice of the parents and also helps in the isolation of suitable germplasm for further improvement. General combining ability is primarily a function of additive and additive $\mathrm{x}$ additive gene action.

Perusal of Table 1 revealed that gca effects of all the characters differed over seasons. Variation in gca effects had also been noticed by Singh et al. (2006) in case of bottle gourd. For example parent Pusa Chikani $\left(\mathrm{P}_{9}\right)$ had good gca effects in both the seasons for maximum characters viz., node number of first male flower anthesis, node number of first female flower anthesis, days taken for anthesis of first male flower, days taken for anthesis of first female flower, days to first marketable fruit harvest, node number to first fruit harvest, primary branches per plant, number of fruits per plan, average fruit weight and marketable fruit yield per plant. The parent $P_{1}$ (NDSG-1) showed good general combining ability for node number of first male flower, node number of first female flower, days for anthesis first female flower, days to first marketable fruit harvest, node number to first fruit harvest, internodal length, fruit diameter, number of fruits per plant and marketable fruit yield per plant during both the years. The parent $\mathrm{P}_{4}$ (NDSG-4) had good general combining ability for node number of first male flower anthesis, node number of first female flower anthesis, days taken for anthesis of first male flower, days to first marketable fruit harvest, node number to first fruit harvest, primary branches per plant and average fruit weight during both the years. Parent NDSG $\left(\mathrm{P}_{8}\right)$ found good general combiner for node number of first male flower, days for anthesis first male flower, primary branches per plant, nodes per vine, vine length, number of fruits per plant and marketable fruit yield per plant during both the years. Such variation may be due to differences in genotypic constitution of the parents and for different characters. Similar results had also been reported by Virendra $e t$ al. (2010).

The ranking of desirable parents on the basis of gca effects for fifteen characters revealed that it was difficult to pickup a single good combiner for all the traits. However, parents $\mathrm{P}_{9}$ (Pusa Chikani), $\mathrm{P}_{4}$ (NDSG-4), $\mathrm{P}_{1}$ (NDSG1), $\mathrm{P}_{2}$ (NDSG-2), $\mathrm{P}_{3}$ (NDSG-3) and $\mathrm{P}_{8}$ (NDSG-8) were found as good general combiners for number of fruits per plant and fruit yield per plant. Parent $\mathrm{P}_{9}$ and $\mathrm{P}_{4}$ also emerged as good general combiner for all the 
maturity traits (early maturity). This show that parents having good gca effects for yield also had good gca effects for one or more yield components. Further, the inbred mentioned above may serve as valuable donors for hybridization programme and may be choosen as most preferred parents for breeding of early maturity high yielding hybrids with good quality in sponge gourd.

Some other parents were also identified as good general combiner for rest of the traits studies other than yield and maturity traits.

In general, the parents which gave the best per se performance were also the best general combiners indicating a positive association between the two parameters. Commonness of parents both for gca effect and per se performance suggested that mean value of parents for a particular traits may have some predictive significance of its gca effect for that trait.

Thus, parents $\mathrm{P}_{4}$ and $\mathrm{P}_{9}$ emerged as the most useful parent as either one or both of them were found good general combiner along with high per se performance for most of the traits studied. Hence, these two parents may also be recommended for exploitation in hybridization programme aimed at improving the yield components for which they were good general combiner.

Table.1 Estimates of gca effects of parents in $9 \times 9$ diallel cross of sponge gourd over two years $\left(\mathrm{Y}_{1}, \mathrm{Y}_{2}\right)$

\begin{tabular}{|c|c|c|c|c|c|c|c|c|c|c|c|c|c|c|}
\hline \multirow[b]{2}{*}{ Parents } & \multicolumn{2}{|c|}{$\begin{array}{l}\text { Node No. of } \\
\text { First Male } \\
\text { Flower }\end{array}$} & \multicolumn{2}{|c|}{$\begin{array}{l}\text { Node No. of } \\
\text { First Female } \\
\text { Flower }\end{array}$} & \multicolumn{2}{|c|}{$\begin{array}{l}\text { Days for Anthesis } \\
\text { First Male } \\
\text { Flower }\end{array}$} & \multicolumn{2}{|c|}{$\begin{array}{c}\text { Days for } \\
\text { Anthesis First } \\
\text { Female Flower }\end{array}$} & \multicolumn{2}{|c|}{$\begin{array}{l}\text { Days to First } \\
\text { Marketable } \\
\text { Fruit harvest }\end{array}$} & \multicolumn{2}{|c|}{$\begin{array}{l}\text { Node No. to } \\
\text { First Fruit } \\
\text { harvest }\end{array}$} & \multicolumn{2}{|c|}{$\begin{array}{c}\text { Primary } \\
\text { Branches/ } \\
\text { Plant }\end{array}$} \\
\hline & $\mathbf{Y}_{1}$ & $\mathbf{Y}_{2}$ & $\mathbf{Y}_{1}$ & $\mathbf{Y}_{2}$ & $\mathbf{Y}_{1}$ & $\mathbf{Y}_{2}$ & $\mathbf{Y}_{1}$ & $\mathbf{Y}_{2}$ & $\mathbf{Y}_{1}$ & $\mathbf{Y}_{2}$ & $\mathbf{Y}_{1}$ & $\mathbf{Y}_{2}$ & $\mathbf{Y}_{1}$ & $\mathbf{Y}_{2}$ \\
\hline NDSG-1 $\left(\mathrm{P}_{1}\right)$ & $-0.62 * *$ & $-0.95 * *$ & $-1.56 * *$ & $-1.10 * *$ & 0.34 & -0.27 & $-1.63 * *$ & $-1.70 * *$ & $-1.75 * *$ & $-2.08 * *$ & $-1.44 * *$ & $-1.08 * *$ & -0.28 & $-0.34 * *$ \\
\hline NDSG-2 $\left(\mathrm{P}_{2}\right)$ & $1.45 * *$ & $1.64 * *$ & $0.70 * *$ & $0.53^{* *}$ & $1.82 * *$ & $1.38^{* *}$ & $1.50 * *$ & $1.59 * *$ & 1.11 & 0.70 & $0.73 * *$ & $0.55 * *$ & -0.29 & $-0.66 * *$ \\
\hline NDSG-3 $\left(\mathrm{P}_{3}\right)$ & $0.34 * *$ & $0.32 * *$ & $0.37 * *$ & $0.32 * *$ & $-0.84^{*}$ & -0.01 & -0.18 & -0.12 & $1.66 * *$ & $1.95^{* *}$ & $0.41 * *$ & $0.26^{* *}$ & -1.22 & $-1.05 * *$ \\
\hline NDSG-4 $\left(\mathrm{P}_{4}\right)$ & $-0.95 * *$ & $-1.15^{* *}$ & $-0.57 * *$ & $-0.53 * *$ & $-1.49 * *$ & $-1.25^{* *}$ & $-1.31 *$ & -1.07 & $-1.29 *$ & $-2.31 * *$ & $-0.70 * *$ & $-0.53 * *$ & 0.60 & $0.18 * *$ \\
\hline NDSG-5 $\left(\mathrm{P}_{5}\right)$ & $-0.60 * *$ & $-0.63 * *$ & $1.53 * *$ & $1.15 * *$ & -0.28 & -0.14 & $1.75^{* *}$ & $1.32 *$ & 1.07 & $1.87 * *$ & $1.50 * *$ & $1.17 * *$ & -0.60 & $-0.62 * *$ \\
\hline NDSG-6 $\left(\mathrm{P}_{6}\right)$ & $2.44 * *$ & $2.54 * *$ & $0.22 *$ & $0.23 * *$ & $1.69 * *$ & $0.93 * *$ & 0.11 & -0.15 & 0.19 & 0.27 & 0.12 & $0.23 *$ & 0.51 & $0.63 * *$ \\
\hline NDSG-7 $\left(\mathrm{P}_{7}\right)$ & $0.55^{* *}$ & $0.54 * *$ & $2.42 * *$ & $2.06 * *$ & $2.08 * *$ & $2.32 * *$ & $2.86^{* *}$ & $2.59 * *$ & $2.80 * *$ & $2.30 * *$ & $2.37 * *$ & $1.96^{* *}$ & -0.12 & -0.01 \\
\hline NDSG-8 $\left(\mathrm{P}_{8}\right)$ & $-1.01 * *$ & $-0.82 * *$ & $0.45 * *$ & $0.45 * *$ & $-2.22 * *$ & $-1.53 * *$ & -0.95 & -0.93 & -1.11 & -0.11 & $0.48 * *$ & $0.51 * *$ & 0.33 & $0.41 * *$ \\
\hline Pusa Chikani & $-1.60 * *$ & $-1.49 * *$ & $-3.56 * *$ & $-3.11 * *$ & $-1.11 * *$ & $-1.42 * *$ & $-2.15 * *$ & $-1.53 * *$ & $-2.69 * *$ & $-2.58 * *$ & $-3.34 * *$ & $-3.07 * *$ & 1.08 & $1.45 * *$ \\
\hline SE Gi & 0.17 & 0.17 & 0.22 & 0.21 & 0.81 & 0.81 & 1.30 & 1.33 & 1.40 & 1.41 & 0.22 & 0.21 & 0.11 & 0.12 \\
\hline SE Gi-Gj & 0.25 & 0.26 & 0.32 & 0.31 & 1.21 & 1.21 & 1.96 & 2.90 & 2.10 & 2.11 & 0.33 & 0.32 & 0.16 & 0.17 \\
\hline
\end{tabular}

\footnotetext{
*, ** Significant at 5 per cent and 1 per cent probability levels, respectively.
} 
Table.1 Contd..

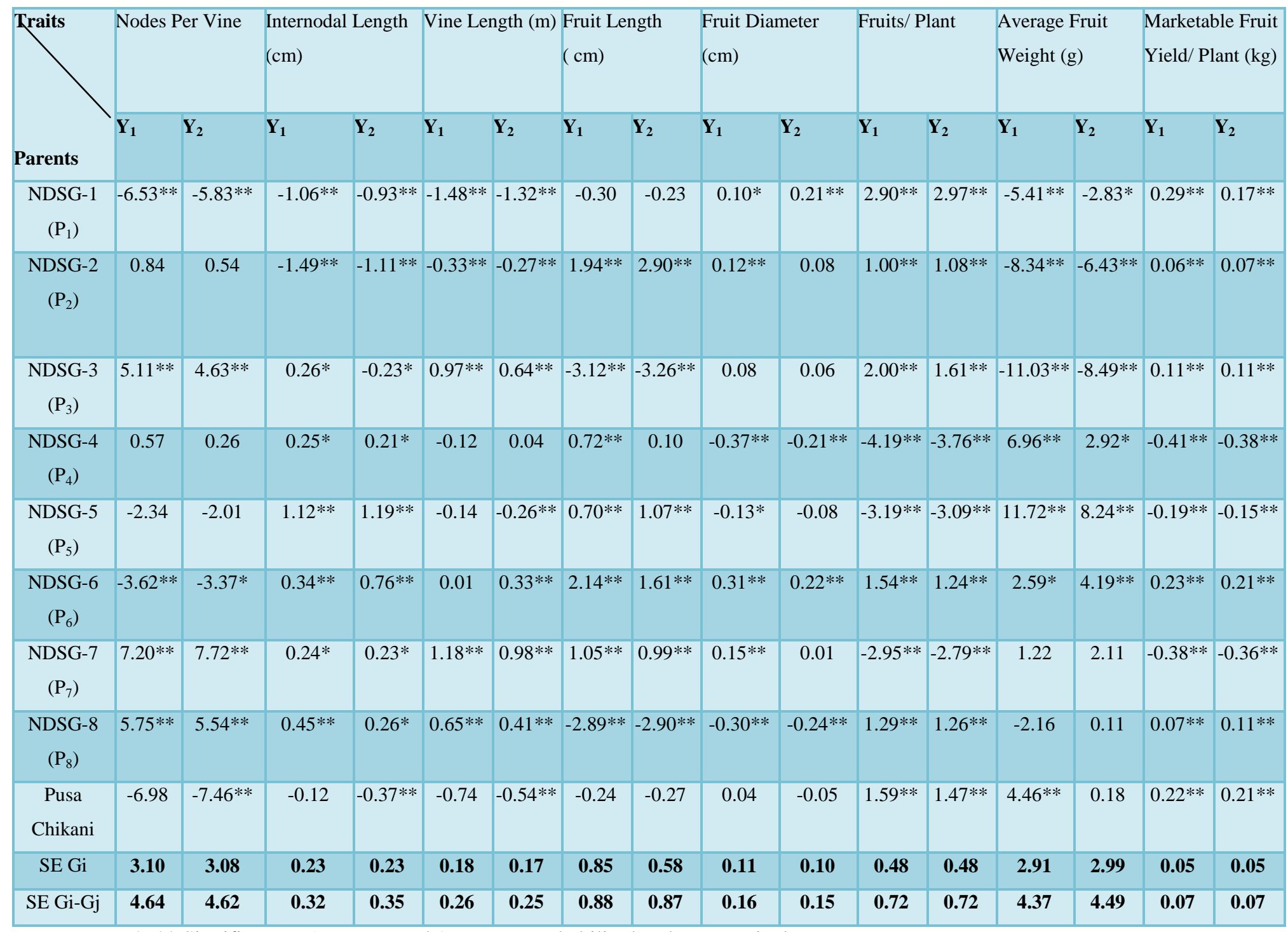

*, ** Significant at 5 per cent and 1 per cent probability levels, respectively. 
Table.2 Estimates of sca effects of parents in $9 \times 9$ diallel cross of sponge gourd over two years $(\mathrm{Y} 1, \mathrm{Y} 2)$

\begin{tabular}{|c|c|c|c|c|c|c|}
\hline Traits & $\begin{array}{l}\text { Node No. of Fi } \\
\text { Flower }\end{array}$ & ist Male & $\begin{array}{l}\text { Node No. of Fi } \\
\text { Flower }\end{array}$ & st Female & $\begin{array}{l}\text { Days for Anthe } \\
\text { Flower }\end{array}$ & is First Male \\
\hline Crosses & $Y_{1}$ & $\mathbf{Y}_{2}$ & $\mathbf{Y}_{1}$ & $\mathbf{Y}_{2}$ & $\mathbf{Y}_{1}$ & $\mathbf{Y}_{2}$ \\
\hline $\mathrm{P}_{1} \times \mathrm{P}_{2}$ & $-1.42^{* *}$ & $-1.33^{* *}$ & $-0.82^{* *}$ & -0.17 & $-3.12^{* *}$ & $-3.52^{* *}$ \\
\hline $\mathrm{P}_{1} \times \mathrm{P}_{3}$ & $0.59^{*}$ & $0.86^{* *}$ & -0.33 & -0.20 & 0.54 & 0.98 \\
\hline $\mathrm{P}_{1} \times \mathrm{P}_{4}$ & $-0.62^{*}$ & $-0.67^{\star *}$ & $1.34^{* *}$ & $1.00^{\star *}$ & 1.19 & 0.12 \\
\hline $\mathrm{P}_{1} \times \mathrm{P}_{5}$ & -0.38 & -0.19 & $-2.31^{* *}$ & $-2.04^{* *}$ & 0.98 & 0.00 \\
\hline $\mathrm{P}_{1} \times \mathrm{P}_{6}$ & $-2.22^{\star *}$ & $-1.76^{* *}$ & $-0.68^{*}$ & $-0.90^{* *}$ & 0.01 & -0.06 \\
\hline $\mathrm{P}_{1} \times \mathrm{P}_{7}$ & -0.32 & 0.25 & $-2.62^{* *}$ & $-1.87^{* *}$ & 0.62 & $2.55^{*}$ \\
\hline $\mathrm{P}_{1} \times \mathrm{P}_{8}$ & -0.36 & $-1.20^{* *}$ & -0.28 & -0.09 & 1.92 & $2.39^{*}$ \\
\hline $\mathrm{P}_{1} \times \mathrm{PC}$ & 0.32 & -0.22 & $1.03^{* *}$ & $-0.84^{* *}$ & -2.19 & -0.71 \\
\hline $\mathrm{P}_{2} \times \mathrm{P}_{3}$ & $-2.13^{* *}$ & $-1.99^{* *}$ & $-1.58^{* *}$ & $-2.43^{* *}$ & -3.94 & $-4.77^{* *}$ \\
\hline $\mathrm{P}_{2} \times \mathrm{P}_{4}$ & $-2.49^{* *}$ & $-2.46^{* *}$ & $-1.25^{* *}$ & $-0.77^{\star \star}$ & 2.70 & $4.47^{* *}$ \\
\hline $\mathrm{P}_{2} \times \mathrm{P}_{5}$ & $1.79^{* *}$ & $1.91^{* *}$ & $3.00^{* *}$ & $2.70^{* *}$ & $-4.50^{* *}$ & $-2.64^{*}$ \\
\hline $\mathrm{P}_{2} \times \mathrm{P}_{6}$ & 0.42 & $0.85^{* *}$ & $-0.73^{* *}$ & 0.46 & $4.52^{* *}$ & $3.29^{* *}$ \\
\hline $\mathrm{P}_{2} \times \mathrm{P}_{7}$ & 1.31 & $1.35^{* *}$ & $-1.19^{* *}$ & $-2.13^{* *}$ & $-2.87^{*}$ & -2.10 \\
\hline $\mathrm{P}_{2} \times \mathrm{P}_{8}$ & $-1.13^{* *}$ & $-0.50^{*}$ & $-2.56^{* *}$ & $-1.76^{* *}$ & $-3.57^{* *}$ & -2.26 \\
\hline $\mathrm{P}_{2} \times \mathrm{PC}$ & $-1.84^{* *}$ & $-2.12^{* *}$ & $-1.13^{* *}$ & $-1.05^{\star *}$ & $3.33^{* *}$ & $2.64^{*}$ \\
\hline $\mathrm{P}_{3} \times \mathrm{P}_{4}$ & $-0.07^{* *}$ & $-0.73^{* *}$ & 0.38 & $1.64^{* *}$ & -0.52 & -0.02 \\
\hline $\mathrm{P}_{3} \times \mathrm{P}_{5}$ & 0.07 & $0.64^{* *}$ & $-1.52^{* *}$ & -0.04 & 0.16 & 0.75 \\
\hline $\mathrm{P}_{3} \times \mathrm{P}_{6}$ & $1.86^{* *}$ & $2.27^{* *}$ & -0.40 & $-1.33^{* *}$ & $-2.81^{*}$ & -1.32 \\
\hline $\mathrm{P}_{3} \times \mathrm{P}_{7}$ & $1.02^{\star *}$ & $1.84^{* *}$ & $-1.60^{* *}$ & $-1.96^{* *}$ & 1.93 & 1.43 \\
\hline $\mathrm{P}_{3} \times \mathrm{P}_{8}$ & $-1.12^{* *}$ & $-1.37^{* *}$ & $3.37^{* *}$ & $2.45^{\star *}$ & -1.90 & -1.86 \\
\hline $\mathrm{P}_{3} \times \mathrm{PC}$ & $-0.83^{\star *}$ & $-0.69^{* *}$ & $-1.32^{* *}$ & $-0.98^{\star *}$ & 1.11 & -0.85 \\
\hline $\mathrm{P}_{4} \times \mathrm{P}_{5}$ & 0.16 & $0.91^{* *}$ & 0.22 & -0.38 & -0.19 & -0.01 \\
\hline $\mathrm{P}_{4} \times \mathrm{P}_{6}$ & $0.92^{* *}$ & -0.05 & $-0.67^{*}$ & -0.17 & $-5.06^{* *}$ & $-3.97^{* *}$ \\
\hline $\mathrm{P}_{4} \times \mathrm{P}_{7}$ & $-1.49^{* *}$ & $-1.15^{* *}$ & 0.33 & -0.30 & $-2.56^{*}$ & -1.47 \\
\hline $\mathrm{P}_{4} \times \mathrm{P}_{8}$ & $1.27^{\star *}$ & $2.10^{* *}$ & $-1.20^{* *}$ & -0.39 & 1.86 & 1.50 \\
\hline $\mathrm{P}_{4} \times \mathrm{PC}$ & $0.56^{*}$ & $0.78^{* *}$ & 0.21 & -0.02 & -1.47 & -0.84 \\
\hline $\mathrm{P}_{5} \times \mathrm{P}_{6}$ & $-1.74^{* *}$ & $-1.68^{* *}$ & 0.43 & -0.15 & 2.63 & 1.81 \\
\hline $\mathrm{P}_{5} \times \mathrm{P}_{7}$ & -0.45 & $-1.08^{* *}$ & $1.23^{* *}$ & $1.12^{* *}$ & 1.51 & -0.32 \\
\hline $\mathrm{P}_{5} \times \mathrm{P}_{8}$ & -0.09 & -0.33 & $-0.80^{*}$ & $-1.37^{* *}$ & -1.35 & -1.62 \\
\hline $\mathrm{P}_{5} \times \mathrm{PC}$ & 0.30 & $0.65^{* *}$ & $-2.49^{* *}$ & $-1.70^{* *}$ & -0.45 & 0.28 \\
\hline $\mathrm{P}_{6} \times \mathrm{P}_{7}$ & $-1.18^{* *}$ & $-0.94^{* *}$ & -0.46 & $0.93^{* *}$ & 1.26 & 0.35 \\
\hline $\mathrm{P}_{6} \times \mathrm{P}_{8}$ & $0.98^{* *}$ & $0.51^{*}$ & $1.52^{* *}$ & 0.54 & $-4.44^{* *}$ & $-3.80^{* *}$ \\
\hline $\mathrm{P}_{6} \times \mathrm{PC}$ & $-2.54^{\star *}$ & $-1.92^{* *}$ & -0.57 & -0.59 & 0.58 & 0.22 \\
\hline $\mathrm{P}_{7} \times \mathrm{P}_{8}$ & $-1.23^{* *}$ & $-1.39^{* *}$ & $2.31^{* *}$ & $1.81^{* *}$ & 1.17 & -0.19 \\
\hline $\mathrm{P}_{7} \times \mathrm{PC}$ & $-0.74^{*}$ & $-0.60^{* *}$ & $-2.68^{* *}$ & $-1.82^{* *}$ & -0.81 & -0.17 \\
\hline $\mathrm{P}_{8} \times \mathrm{PC}$ & $1.02^{* *}$ & $0.84^{* *}$ & $-1.00^{* *}$ & $-1.11^{* *}$ & -0.63 & 0.55 \\
\hline SE Sij & 0.47 & 0.49 & 0.61 & 0.59 & 2.29 & 2.29 \\
\hline SE Sij-Skl & 0.66 & 0.72 & 0.90 & 0.87 & 3.38 & 3.37 \\
\hline
\end{tabular}


Table. 2 contd....

\begin{tabular}{|c|c|c|c|c|c|c|}
\hline Traits & $\begin{array}{l}\text { Days for Anthe } \\
\text { Flower }\end{array}$ & is First Female & $\begin{array}{l}\text { Days to First } \mathrm{N} \\
\text { harvest }\end{array}$ & arketable Friut & Node No. to Fi & st Fruit harvest \\
\hline Crosses & $Y_{1}$ & $\mathbf{Y}_{2}$ & $Y_{1}$ & $\mathbf{Y}_{2}$ & $Y_{1}$ & $\mathbf{Y}_{2}$ \\
\hline $\mathrm{P}_{1} \times \mathrm{P}_{2}$ & -3.21 & -2.91 & $-5.08^{*}$ & -3.58 & -0.27 & -0.13 \\
\hline $\mathrm{P}_{1} \times \mathrm{P}_{3}$ & -0.06 & 2.47 & -2.63 & -3.83 & -0.48 & -0.07 \\
\hline $\mathrm{P}_{1} \times \mathrm{P}_{4}$ & 0.60 & 1.76 & 1.32 & 1.44 & $1.15^{* *}$ & $0.85^{* *}$ \\
\hline $\mathrm{P}_{1} \times \mathrm{P}_{5}$ & $-4.46^{*}$ & -3.64 & -2.05 & -3.75 & $-2.10 * *$ & $-2.00 * *$ \\
\hline $\mathrm{P}_{1} \times \mathrm{P}_{6}$ & 0.18 & 0.84 & 1.83 & 2.85 & $-0.70 * *$ & $-0.84 * *$ \\
\hline $\mathrm{P}_{1} \times \mathrm{P}_{7}$ & -1.57 & -0.91 & 0.23 & 1.83 & $-2.29 * *$ & $-1.91 * *$ \\
\hline $\mathrm{P}_{1} \times \mathrm{P}_{8}$ & 4.24 & 2.61 & 2.13 & 2.24 & -0.02 & 0.01 \\
\hline $\mathrm{P}_{1} \times \mathrm{PC}$ & 2.44 & 0.21 & 2.71 & 1.00 & $0.92 * *$ & $-0.72 *$ \\
\hline $\mathrm{P}_{2} \times \mathrm{P}_{3}$ & $-7.67 * *$ & $-7.49 * *$ & 0.92 & -1.57 & $-1.14 * *$ & $-2.20 * *$ \\
\hline $\mathrm{P}_{2} \times \mathrm{P}_{4}$ & 2.47 & 2.46 & 0.46 & 0.66 & $-1.34 * *$ & $-0.91 * *$ \\
\hline $\mathrm{P}_{2} \times \mathrm{P}_{5}$ & 2.41 & 1.07 & 1.10 & -0.52 & $2.81 * *$ & $2.54 * *$ \\
\hline $\mathrm{P}_{2} \times \mathrm{P}_{6}$ & -0.96 & -0.46 & -2.02 & -0.92 & $-0.86^{* *}$ & $0.63^{*}$ \\
\hline $\mathrm{P}_{2} \times \mathrm{P}_{7}$ & 2.23 & 0.71 & 1.37 & 1.05 & $-1.37 * *$ & $-2.16^{* *}$ \\
\hline $\mathrm{P}_{2} \times \mathrm{P}_{8}$ & 1.10 & -0.69 & 3.28 & 1.46 & $-2.31 * *$ & $-1.35 * *$ \\
\hline $\mathrm{P}_{2} \times \mathrm{PC}$ & 0.31 & 0.92 & -0.14 & -1.08 & $-1.25 * *$ & $-1.23 * *$ \\
\hline $\mathrm{P}_{3} \times \mathrm{P}_{4}$ & 1.15 & 2.17 & 1.92 & 1.41 & 0.28 & $1.57 * *$ \\
\hline $\mathrm{P}_{3} \times \mathrm{P}_{5}$ & 0.09 & -1.22 & 0.55 & -0.78 & $-1.72 * *$ & -0.12 \\
\hline $\mathrm{P}_{3} \times \mathrm{P}_{6}$ & 0.72 & 1.26 & 1.43 & 1.82 & -0.54 & $-1.38 * *$ \\
\hline $\mathrm{P}_{3} \times \mathrm{P}_{7}$ & 2.97 & 1.51 & 0.82 & -0.20 & $-1.59 * *$ & $-1.91 * *$ \\
\hline $\mathrm{P}_{3} \times \mathrm{P}_{8}$ & -0.22 & -1.97 & -1.27 & -1.79 & $3.10 * *$ & $2.34 * *$ \\
\hline $\mathrm{P}_{3} \times \mathrm{PC}$ & $-5.01 * *$ & $-4.37 *$ & -3.69 & -3.33 & $-1.15^{* *}$ & $-1.08 * *$ \\
\hline $\mathrm{P}_{4} \times \mathrm{P}_{5}$ & 2.22 & 2.73 & 0.50 & 1.49 & 0.19 & -0.33 \\
\hline $\mathrm{P}_{4} \times \mathrm{P}_{6}$ & -1.14 & -0.79 & -1.62 & -1.91 & $-0.64 *$ & -0.29 \\
\hline $\mathrm{P}_{4} \times \mathrm{P}_{7}$ & -0.90 & 0.46 & 1.77 & 2.66 & $0.62 *$ & -0.32 \\
\hline $\mathrm{P}_{4} \times \mathrm{P}_{8}$ & -0.08 & 0.98 & -0.32 & -1.23 & $-1.29 * *$ & -0.57 \\
\hline $\mathrm{P}_{4} \times \mathrm{PC}$ & -2.88 & -2.42 & -1.74 & 0.14 & 0.26 & 0.21 \\
\hline $\mathrm{P}_{5} \times \mathrm{P}_{6}$ & 0.80 & 0.12 & 2.01 & 0.01 & 0.36 & -0.08 \\
\hline $\mathrm{P}_{5} \times \mathrm{P}_{7}$ & 1.04 & 2.07 & 1.41 & 2.88 & $1.12 * *$ & $1.09 * *$ \\
\hline $\mathrm{P}_{5} \times \mathrm{P}_{8}$ & $-5.14 * *$ & -3.41 & -3.68 & 2.29 & -0.59 & $-1.17 * *$ \\
\hline $\mathrm{P}_{5} \times \mathrm{PC}$ & 0.06 & 0.19 & -0.11 & -2.25 & $-2.04 * *$ & $-1.68 * *$ \\
\hline $\mathrm{P}_{6} \times \mathrm{P}_{7}$ & $-4.32 *$ & -3.46 & -3.71 & $-4.52 *$ & -0.51 & $0.93 * *$ \\
\hline $\mathrm{P}_{6} \times \mathrm{P}_{8}$ & 0.49 & -0.94 & -0.80 & -1.11 & $1.88 * *$ & $0.67 *$ \\
\hline $\mathrm{P}_{6} \times \mathrm{PC}$ & 0.70 & 0.66 & -0.23 & 0.35 & -0.56 & $-0.64 *$ \\
\hline $\mathrm{P}_{7} \times \mathrm{P}_{8}$ & $-5.26 * *$ & $-4.69 *$ & $-5.41 *$ & $-5.14^{*}$ & $2.14 * *$ & $1.74 * *$ \\
\hline $\mathrm{P}_{7} \times \mathrm{PC}$ & 1.95 & 1.92 & 0.17 & -0.67 & $-2.61 * *$ & $-1.87 * *$ \\
\hline $\mathrm{P}_{8} \times \mathrm{PC}$ & 1.76 & 2.44 & 1.07 & 0.74 & $-1.02 * *$ & $-1.12 * *$ \\
\hline SE Sij & 3.69 & 3.76 & 3.97 & 3.98 & 0.62 & 0.60 \\
\hline SE Sij-Skl & 5.44 & 5.54 & 5.85 & 5.87 & 0.91 & 0.88 \\
\hline
\end{tabular}


Table. 2 contd....

\begin{tabular}{|c|c|c|c|c|c|c|}
\hline Traits & Primary Brancl & es/Plant & Nodes Per Vine & & Internodal Len & th $(\mathrm{cm})$ \\
\hline Crosses & $\mathbf{Y}_{1}$ & $\mathbf{Y}_{2}$ & $\mathbf{Y}_{1}$ & $\mathbf{Y}_{2}$ & $\mathbf{Y}_{1}$ & $\mathbf{Y}_{2}$ \\
\hline $\mathrm{P}_{1} \times \mathrm{P}_{2}$ & $0.54 * *$ & -0.18 & -0.69 & 1.18 & 0.17 & 0.20 \\
\hline $\mathrm{P}_{1} \times \mathrm{P}_{3}$ & $1.48 * *$ & -0.10 & 0.04 & -1.91 & 0.42 & -0.29 \\
\hline $\mathrm{P}_{1} \times \mathrm{P}_{4}$ & $0.99 * *$ & $0.88 * *$ & $17.58 * *$ & $15.45^{* *}$ & $-1.91 * *$ & $-1.32 * *$ \\
\hline $\mathrm{P}_{1} \times \mathrm{P}_{5}$ & $0.85^{* *}$ & $0.87 * *$ & 2.49 & 4.73 & -0.11 & -0.10 \\
\hline $\mathrm{P}_{1} \times \mathrm{P}_{6}$ & -0.26 & $-0.78 * *$ & -2.24 & -0.91 & 0.01 & 0.22 \\
\hline $\mathrm{P}_{1} \times \mathrm{P}_{7}$ & $-0.62 * *$ & -0.13 & $8.95^{*}$ & 7.00 & $1.11 * *$ & $1.86^{* *}$ \\
\hline $\mathrm{P}_{1} \times \mathrm{P}_{8}$ & $-0.88 * *$ & $-0.46^{* *}$ & -4.60 & -2.82 & -0.10 & -0.37 \\
\hline $\mathrm{P}_{1} \times \mathrm{PC}$ & $-1.83 * *$ & $-1.29 * *$ & 5.13 & 4.18 & -0.20 & -0.44 \\
\hline $\mathrm{P}_{2} \times \mathrm{P}_{3}$ & $-0.51 * *$ & -0.08 & -5.33 & -5.27 & 0.44 & 0.31 \\
\hline $\mathrm{P}_{2} \times \mathrm{P}_{4}$ & $0.97 * *$ & $0.59 * *$ & 8.22 & 8.09 & 0.52 & 0.16 \\
\hline $\mathrm{P}_{2} \times \mathrm{P}_{5}$ & -0.14 & -0.21 & 6.13 & 5.36 & -0.34 & 0.38 \\
\hline $\mathrm{P}_{2} \times \mathrm{P}_{6}$ & -0.25 & $-0.46^{* *}$ & 5.40 & 4.73 & $-1.57 * *$ & $-0.96^{* *}$ \\
\hline $\mathrm{P}_{2} \times \mathrm{P}_{7}$ & $1.18 * *$ & -0.22 & -3.42 & -1.36 & $-2.44 * *$ & $-1.46^{* *}$ \\
\hline $\mathrm{P}_{2} \times \mathrm{P}_{8}$ & $-1.07 * *$ & 0.06 & $15.04 * *$ & $13.82 * *$ & $0.99 * *$ & 0.00 \\
\hline $\mathrm{P}_{2} \times \mathrm{PC}$ & 0.18 & 0.23 & 4.76 & 3.82 & $-2.10 * *$ & $-1.36 * *$ \\
\hline $\mathrm{P}_{3} \times \mathrm{P}_{4}$ & $0.61 * *$ & $0.40^{*}$ & $18.95^{* *}$ & $19.00^{* *}$ & $0.82 *$ & 0.57 \\
\hline $\mathrm{P}_{3} \times \mathrm{P}_{5}$ & -0.21 & $0.48 * *$ & $-11.15^{* *}$ & -9.73 & $2.24 * *$ & $1.20 * *$ \\
\hline $\mathrm{P}_{3} \times \mathrm{P}_{6}$ & $0.69 * *$ & $0.63 * *$ & $35.13 * *$ & $35.64 * *$ & $-1.01 * *$ & -0.28 \\
\hline $\mathrm{P}_{3} \times \mathrm{P}_{7}$ & $0.33^{*}$ & 0.28 & -7.69 & -6.45 & -0.21 & -0.61 \\
\hline $\mathrm{P}_{3} \times \mathrm{P}_{8}$ & $-0.34 *$ & $-0.56^{* *}$ & $24.76^{* *}$ & $23.73 * *$ & 0.58 & 0.14 \\
\hline $\mathrm{P}_{3} \times \mathrm{PC}$ & $-0.88 * *$ & $1.14 * *$ & -1.51 & -2.27 & $-0.84^{*}$ & -0.45 \\
\hline $\mathrm{P}_{4} \times \mathrm{P}_{5}$ & -0.03 & $-0.55^{* *}$ & $25.40 * *$ & $23.64 * *$ & $-1.08 * *$ & $0.66^{*}$ \\
\hline $\mathrm{P}_{4} \times \mathrm{P}_{6}$ & -0.12 & $1.02 * *$ & -7.33 & -5.00 & -0.31 & $-0.72 *$ \\
\hline $\mathrm{P}_{4} \times \mathrm{P}_{7}$ & $-1.50 * *$ & $-0.66^{* *}$ & $-16.15^{* *}$ & $-18.09 * *$ & $1.79 * *$ & $0.76^{*}$ \\
\hline $\mathrm{P}_{4} \times \mathrm{P}_{8}$ & $-0.95 * *$ & $-1.46^{* *}$ & -3.69 & -1.91 & $-1.42 * *$ & $-0.91 * *$ \\
\hline $\mathrm{P}_{4} \times \mathrm{PC}$ & $0.46^{*}$ & 0.06 & -1.96 & -1.91 & $2.22 * *$ & $1.32 * *$ \\
\hline $\mathrm{P}_{5} \times \mathrm{P}_{6}$ & 0.06 & $-0.50 * *$ & 7.58 & 6.27 & $-1.18^{* *}$ & -0.50 \\
\hline $\mathrm{P}_{5} \times \mathrm{P}_{7}$ & -0.28 & -0.13 & $8.76^{*}$ & 7.18 & $-1.11 * *$ & $-2.23 * *$ \\
\hline $\mathrm{P}_{5} \times \mathrm{P}_{8}$ & 0.25 & $0.34^{*}$ & 4.22 & 2.36 & $0.86^{*}$ & -0.29 \\
\hline $\mathrm{P}_{5} \times \mathrm{PC}$ & 0.50 & $-0.99 * *$ & 3.95 & 6.36 & $2.84 * *$ & $1.34 * *$ \\
\hline $\mathrm{P}_{6} \times \mathrm{P}_{7}$ & $-0.42^{*}$ & $-1.11 * *$ & $12.04 * *$ & $9.55^{*}$ & -0.30 & 0.97 \\
\hline $\mathrm{P}_{6} \times \mathrm{P}_{8}$ & $0.33 *$ & -0.24 & $-10.51 * *$ & $-12.27 * *$ & -0.84 & -0.07 \\
\hline $\mathrm{P}_{6} \times \mathrm{PC}$ & $-0.60 * *$ & 0.16 & -3.78 & -5.27 & 0.18 & 0.06 \\
\hline $\mathrm{P}_{7} \times \mathrm{P}_{8}$ & $0.86^{* *}$ & $0.40 *$ & $12.67 * *$ & 15.64 & 0.59 & $0.97 * *$ \\
\hline $\mathrm{P}_{7} \times \mathrm{PC}$ & 0.03 & $-0.70 * *$ & $26.40 * *$ & $25.64 * *$ & $-2.83^{* *}$ & $-1.64 * *$ \\
\hline $\mathrm{P}_{8} \times \mathrm{PC}$ & $0.55 * *$ & -0.05 & 1.85 & 3.82 & $1.96 * *$ & $1.27 * *$ \\
\hline SE Sij & 0.31 & 0.33 & 8.77 & 8.73 & 0.65 & 0.66 \\
\hline SE Sij-Skl & 0.45 & 0.48 & 12.93 & 12.87 & 0.96 & 0.97 \\
\hline
\end{tabular}


Table. 2 contd....

\begin{tabular}{|c|c|c|c|c|c|c|}
\hline Traits & Vine Length & & Fruit Length ( & & Fruit Diameter & $\mathrm{cm})$ \\
\hline & $\mathbf{Y}_{1}$ & $\mathbf{Y}_{2}$ & $\mathbf{Y}_{1}$ & $\mathbf{Y}_{2}$ & $\mathbf{Y}_{1}$ & $\mathbf{Y}_{2}$ \\
\hline $\mathrm{P}_{1} \times \mathrm{P}_{2}$ & $-0.51 *$ & 0.01 & -0.11 & 0.49 & 0.00 & 0.25 \\
\hline $\mathrm{P}_{1} \times \mathrm{P}_{3}$ & $-0.63 *$ & $-0.62 *$ & -0.06 & 1.24 & -0.17 & 0.06 \\
\hline $\mathrm{P}_{1} \times \mathrm{P}_{4}$ & 0.38 & 0.30 & 0.20 & 0.09 & 0.29 & $-0.31 *$ \\
\hline $\mathrm{P}_{1} \times \mathrm{P}_{5}$ & $-1.10 * *$ & $-0.64 * *$ & -1.11 & 0.12 & -0.02 & -0.18 \\
\hline $\mathrm{P}_{1} \times \mathrm{P}_{6}$ & 0.44 & 0.39 & -0.21 & -1.43 & -0.07 & 0.00 \\
\hline $\mathrm{P}_{1} \times \mathrm{P}_{7}$ & $-1.31 * *$ & $-0.72 * *$ & -0.13 & -1.10 & -0.01 & -0.08 \\
\hline $\mathrm{P}_{1} \times \mathrm{P}_{8}$ & 0.22 & 0.03 & $2.12 *$ & $2.82 * *$ & -0.06 & 0.25 \\
\hline $\mathrm{P}_{1} \times \mathrm{PC}$ & 2.61 & $1.58 * *$ & $-1.93 *$ & $-2.05^{*}$ & 0.10 & -0.03 \\
\hline $\mathrm{P}_{2} \times \mathrm{P}_{3}$ & 0.33 & $0.53 *$ & -1.19 & -0.38 & -0.06 & -0.21 \\
\hline $\mathrm{P}_{2} \times \mathrm{P}_{4}$ & 0.52 & 0.03 & -0.03 & 1.27 & -0.21 & -0.16 \\
\hline $\mathrm{P}_{2} \times \mathrm{P}_{5}$ & -0.04 & 0.16 & 0.72 & 0.63 & 0.05 & -0.16 \\
\hline $\mathrm{P}_{2} \times \mathrm{P}_{6}$ & -0.31 & -0.36 & -0.45 & -0.02 & -0.21 & -0.17 \\
\hline $\mathrm{P}_{2} \times \mathrm{P}_{7}$ & -0.36 & -0.04 & 0.30 & -0.40 & -0.06 & 0.00 \\
\hline $\mathrm{P}_{2} \times \mathrm{P}_{8}$ & $0.95 * *$ & $0.75 * *$ & 1.58 & -0.33 & 0.11 & 0.00 \\
\hline $\mathrm{P}_{2} \times \mathrm{PC}$ & $0.96 * *$ & $0.63^{*}$ & -0.17 & 0.03 & -0.01 & -0.05 \\
\hline $\mathrm{P}_{3} \times \mathrm{P}_{4}$ & $2.22 * *$ & $1.62 * *$ & 1.00 & $-2.91 * *$ & 0.14 & 0.18 \\
\hline $\mathrm{P}_{3} \times \mathrm{P}_{5}$ & $-1.46^{* *}$ & $-1.48 * *$ & $3.47 * *$ & $3.32 * *$ & 0.00 & -0.10 \\
\hline $\mathrm{P}_{3} \times \mathrm{P}_{6}$ & $2.49 * *$ & $2.03 * *$ & 0.50 & 0.90 & -0.24 & 0.05 \\
\hline $\mathrm{P}_{3} \times \mathrm{P}_{7}$ & $1.11 * *$ & $0.48^{*}$ & -1.24 & $-1.70^{*}$ & 0.22 & 0.26 \\
\hline $\mathrm{P}_{3} \times \mathrm{P}_{8}$ & $2.85 * *$ & $2.65 * *$ & -1.10 & 0.85 & $0.37 *$ & 0.24 \\
\hline $\mathrm{P}_{3} \times \mathrm{PC}$ & -0.46 & $-0.51 *$ & 0.96 & 0.73 & -0.07 & -0.09 \\
\hline $\mathrm{P}_{4} \times \mathrm{P}_{5}$ & $1.73 * *$ & $2.02 * *$ & -0.74 & -0.36 & -0.01 & -0.03 \\
\hline $\mathrm{P}_{4} \times \mathrm{P}_{6}$ & $-1.18 * *$ & $-0.67 * *$ & -0.81 & -1.07 & 0.03 & $0.37 *$ \\
\hline $\mathrm{P}_{4} \times \mathrm{P}_{7}$ & $1.71 * *$ & $1.38 * *$ & 0.74 & 1.07 & -0.11 & -0.10 \\
\hline $\mathrm{P}_{4} \times \mathrm{P}_{8}$ & $-0.67 *$ & -0.45 & -0.46 & 0.95 & -0.22 & -0.22 \\
\hline $\mathrm{P}_{4} \times \mathrm{PC}$ & $-1.97 * *$ & $-1.10^{* *}$ & $-1.88^{*}$ & 1.04 & 0.08 & $0.38^{*}$ \\
\hline $\mathrm{P}_{5} \times \mathrm{P}_{6}$ & 0.20 & 0.32 & 0.68 & 0.27 & 0.15 & $-0.31 *$ \\
\hline $\mathrm{P}_{5} \times \mathrm{P}_{7}$ & $1.73 * *$ & $1.18 * *$ & -1.29 & -0.92 & -0.15 & $-0.44 * *$ \\
\hline $\mathrm{P}_{5} \times \mathrm{P}_{8}$ & $0.76^{*}$ & -0.46 & 0.56 & $-2.13 *$ & -0.12 & -0.19 \\
\hline $\mathrm{P}_{5} \times \mathrm{PC}$ & 0.44 & 0.29 & -0.61 & -0.83 & 0.04 & 0.34 \\
\hline $\mathrm{P}_{6} \times \mathrm{P}_{7}$ & $-2.02 * *$ & $-1.61 * *$ & 0.33 & 0.35 & -0.11 & 0.06 \\
\hline $\mathrm{P}_{6} \times \mathrm{P}_{8}$ & 0.41 & $0.76 * *$ & -0.72 & 1.25 & 0.15 & 0.06 \\
\hline $\mathrm{P}_{6} \times \mathrm{PC}$ & $-0.60 *$ & -0.20 & -0.28 & -0.30 & -0.10 & -0.44 \\
\hline $\mathrm{P}_{7} \times \mathrm{P}_{8}$ & -0.47 & $-0.49 *$ & -0.83 & -1.35 & 0.00 & -0.13 \\
\hline $\mathrm{P}_{7} \times \mathrm{PC}$ & $1.62 * *$ & $1.95 * *$ & $1.81 *$ & 1.32 & -0.14 & -0.23 \\
\hline $\mathrm{P}_{8} \times \mathrm{PC}$ & -0.05 & -0.18 & 0.09 & 0.13 & -0.14 & -0.18 \\
\hline SE Sij & 0.50 & 0.48 & 1.65 & 1.64 & 0.30 & 0.29 \\
\hline SE Sij-Skl & 0.73 & 0.70 & 2.44 & 2.41 & 0.44 & 0.43 \\
\hline
\end{tabular}


Table. 2 contd....

\begin{tabular}{|c|c|c|c|c|c|c|}
\hline Traits & Number of frui & s per plant & Average fruit & weight (g) & $\begin{array}{l}\text { Marketable fr } \\
\text { plant (kg) }\end{array}$ & it yield per \\
\hline Crosses & $Y_{1}$ & $\mathbf{Y}_{2}$ & $\mathbf{Y}_{1}$ & $\mathbf{Y}_{2}$ & $\mathbf{Y}_{1}$ & $\mathbf{Y}_{2}$ \\
\hline $\mathrm{P}_{1} \times \mathrm{P}_{2}$ & 0.99 & $1.92 * *$ & -7.03 & -8.74 & $0.42 * *$ & $0.36^{* *}$ \\
\hline $\mathrm{P}_{1} \times \mathrm{P}_{3}$ & $-2.01 * *$ & -0.61 & $22.91 * *$ & 13.79 & -0.03 & $0.30 * *$ \\
\hline $\mathrm{P}_{1} \times \mathrm{P}_{4}$ & $-2.82 * *$ & -1.25 & -4.61 & -2.44 & $-0.31 * *$ & $-0.45 * *$ \\
\hline $\mathrm{P}_{1} \times \mathrm{P}_{5}$ & $-1.82^{*}$ & $-1.91 * *$ & -0.34 & 1.25 & -0.12 & 0.01 \\
\hline $\mathrm{P}_{1} \times \mathrm{P}_{6}$ & -0.55 & -0.75 & $-10.70^{*}$ & -3.10 & $-0.35^{* *}$ & -0.13 \\
\hline $\mathrm{P}_{1} \times \mathrm{P}_{7}$ & $-1.73^{*}$ & $-2.91 * *$ & -1.43 & -1.04 & $-0.35^{* *}$ & $-0.19 *$ \\
\hline $\mathrm{P}_{1} \times \mathrm{P}_{8}$ & 1.03 & 0.53 & 4.51 & 9.61 & $0.21 * *$ & $0.40 * *$ \\
\hline $\mathrm{P}_{1} \times \mathrm{PC}$ & $1.40^{*}$ & $1.52 *$ & 7.93 & 13.35 & $0.25 * *$ & 0.10 \\
\hline $\mathrm{P}_{2} \times \mathrm{P}_{3}$ & 0.01 & 0.07 & $21.52 * *$ & 12.99 & $0.28 * *$ & $0.27^{* *}$ \\
\hline $\mathrm{P}_{2} \times \mathrm{P}_{4}$ & 0.74 & 1.24 & -3.48 & 1.37 & 0.03 & $-0.16^{*}$ \\
\hline $\mathrm{P}_{2} \times \mathrm{P}_{5}$ & 1.08 & 0.28 & $9.62 *$ & 6.58 & $0.19 * *$ & $0.29 * *$ \\
\hline $\mathrm{P}_{2} \times \mathrm{P}_{6}$ & -0.32 & 0.84 & 0.23 & 0.91 & -0.01 & 0.08 \\
\hline $\mathrm{P}_{2} \times \mathrm{P}_{7}$ & $-2.16^{* *}$ & -1.32 & 5.06 & 5.38 & $-0.21 * *$ & -0.11 \\
\hline $\mathrm{P}_{2} \times \mathrm{P}_{8}$ & 0.93 & 1.13 & 2.98 & 5.88 & $0.15^{*}$ & $0.21 * *$ \\
\hline $\mathrm{P}_{2} \times \mathrm{PC}$ & $-1.70^{*}$ & $-2.39 * *$ & $-11.21 * *$ & -2.82 & $-0.32 * *$ & $-0.38 * *$ \\
\hline $\mathrm{P}_{3} \times \mathrm{P}_{4}$ & $-1.92 * *$ & $-2.68 * *$ & $8.55^{*}$ & 12.74 & -0.12 & $-0.19 * *$ \\
\hline $\mathrm{P}_{3} \times \mathrm{P}_{5}$ & 0.08 & -0.35 & 5.29 & 9.23 & 0.12 & $-0.17 *$ \\
\hline $\mathrm{P}_{3} \times \mathrm{P}_{6}$ & -0.65 & -0.88 & $-15.08 * *$ & -4.43 & $-0.26^{* *}$ & -0.09 \\
\hline $\mathrm{P}_{3} \times \mathrm{P}_{7}$ & $4.84 * *$ & $4.86 * *$ & $-8.72 *$ & -6.45 & $0.37 * *$ & $0.47 * *$ \\
\hline $\mathrm{P}_{3} \times \mathrm{P}_{8}$ & -0.07 & -0.80 & $-10.33^{*}$ & -1.65 & $-0.26^{* *}$ & $-0.30 * *$ \\
\hline $\mathrm{P}_{3} \times \mathrm{PC}$ & $2.63 * *$ & $2.49 * *$ & -1.95 & -10.12 & $0.55^{* *}$ & $0.48^{* *}$ \\
\hline $\mathrm{P}_{4} \times \mathrm{P}_{5}$ & $2.27 * *$ & 1.22 & $-12.21 * *$ & -12.49 & $0.34 * *$ & $0.29 * *$ \\
\hline $\mathrm{P}_{4} \times \mathrm{P}_{6}$ & 0.54 & 0.58 & -0.07 & -9.65 & 0.14 & $0.17 *$ \\
\hline $\mathrm{P}_{4} \times \mathrm{P}_{7}$ & 0.03 & 0.52 & -0.71 & 2.83 & -0.07 & -0.05 \\
\hline $\mathrm{P}_{4} \times \mathrm{P}_{8}$ & -1.21 & $-1.94 * *$ & 7.01 & 0.44 & -0.09 & -0.08 \\
\hline $\mathrm{P}_{4} \times \mathrm{PC}$ & $3.16 * *$ & $3.35 * *$ & -6.94 & -15.83 & $0.36 * *$ & $0.52 * *$ \\
\hline $\mathrm{P}_{5} \times \mathrm{P}_{6}$ & 0.54 & 0.62 & 4.50 & -0.46 & $0.15^{*}$ & $0.21 *$ \\
\hline $\mathrm{P}_{5} \times \mathrm{P}_{7}$ & 1.03 & 0.75 & -3.80 & -2.98 & -0.06 & -0.07 \\
\hline $\mathrm{P}_{5} \times \mathrm{P}_{8}$ & -0.21 & 0.90 & $-13.08 * *$ & -0.88 & -0.10 & -0.15 \\
\hline $\mathrm{P}_{5} \times \mathrm{PC}$ & $-1.51 *$ & -0.41 & 3.96 & 7.75 & $-0.16^{*}$ & -0.06 \\
\hline $\mathrm{P}_{6} \times \mathrm{P}_{7}$ & -0.70 & -1.18 & 4.00 & 0.46 & 0.11 & 0.05 \\
\hline $\mathrm{P}_{6} \times \mathrm{P}_{8}$ & 1.06 & 0.46 & -3.95 & 1.67 & 0.07 & 0.17 \\
\hline $\mathrm{P}_{6} \times \mathrm{PC}$ & $1.76^{*}$ & 1.15 & $10.10 *$ & 9.80 & $0.34 * *$ & 0.24 \\
\hline $\mathrm{P}_{7} \times \mathrm{P}_{8}$ & $3.88 * *$ & $3.10 * *$ & 2.41 & 5.25 & $0.66 * *$ & $0.36 * *$ \\
\hline $\mathrm{P}_{7} \times \mathrm{PC}$ & -0.75 & 0.49 & 1.80 & 5.88 & 0.05 & 0.09 \\
\hline $\mathrm{P}_{8} \times \mathrm{PC}$ & $2.01 * *$ & 1.14 & $-10.82^{*}$ & -22.22 & 0.08 & $0.17 *$ \\
\hline SE Sij & 1.37 & 1.36 & 8.24 & 8.47 & 0.14 & 0.14 \\
\hline SE Sij-Skl & 2.01 & 1.82 & 12.16 & 12.49 & 0.20 & 0.21 \\
\hline
\end{tabular}


The sca effects represent non-additive gene action which is non-fixable. Specific combining ability effects helps in the identification of superior cross combinations for development of promising varieties/ hybrids. The crosses showing high sca effects involving parents with high gca effects may give rise desirable segregants in future generation. The specific combining ability effects of the thirty six crosses for fourteen traits in both the years have been presented in Table 2. Perusal of Table 2 revealed that significant positive and negative sca effects were observed for all the characters. However, none of the crosses had significant sca effect for all the traits. Further, sca effects were found to vary in nature and magnitude for all the characters with the change of seasons. This varying magnitude of sca effects over seasons may be due to environmental effects and genotypes, respectively. Virendra et al. (2010) also reported similar results.

Development of high yielding $F_{1}$ coupled with more number of fruits per plant is an important aspect. Out of ten crosses which showed significant sca effects for marketable fruit yield per plant in either of the seasons, there were only three crosses namely, $\mathrm{P}_{3} \times \mathrm{P}_{7}$, $\begin{array}{lllllllll}\mathrm{P}_{4} & \mathrm{x} & \mathrm{P}_{9} & \text { and } & \mathrm{P}_{7} & \mathrm{x} & \mathrm{P}_{8} & \text { which exhibited }\end{array}$ significant sca effects both for fruit yield and number of fruits per plant over both the seasons. Therefore, these crosses may likely to fit for farmers demand and also throw good transgressive segregates combining more number of fruits coupled with high fruit yield in later generations of selection. There was a close correspondence between sca effect and the heterosis.

Ten cross combinations across the years showed significant and positive specific combining ability effects for fruit yield involving parents with High yield to average $(\mathrm{H} \times \mathrm{A}) \times \mathrm{High}(\mathrm{L} \times \mathrm{H})$, Low $x$ Low $(\mathrm{L} \times \mathrm{L})$,
High $\mathrm{x}$ Low $(\mathrm{H} \times \mathrm{L})$ and High $\mathrm{x} \operatorname{High}(\mathrm{H} \times \mathrm{H})$ general combining ability effects for fruit yield. Similar results have also been reported by Maurya (2010).

Perusal of Table 2 revealed that out of $36 \mathrm{~F}_{1}$ hybrids, significant sca effects in favourable direction were exhibited by fourteen and seventeen hybrids for node number of first male flower anthesis, Eighteen and sixteen hybrids for node number of fist female flower anthesis, nine and five for days taken for anthesis of first male flower, six and three hybrids for days for anthesis of first female flower, two hybrids in each year for days to first marketable fruit harvest, seventeen and fourteen crosses for node number to first fruit harvest, thirteen and eight hybrids for no. of primary branches per vine at last harvest, eleven and seven crosses for nodes per vine and internodal length, eleven and thirteen hybrids for vine length, three and two hybrids for fruit length, one and two hybrids for fruit diameter, eight and six hybrids for number of fruits per plant, five and eight hybrids for average fruit weight, eleven and fourteen hybrids for marketable fruit yield per plant, $\mathrm{Y}_{1}$ and $\mathrm{Y}_{2}$, respectively. Significant sca effects had also been reputed for these traits by earlier scientists like Gupta et al. (2006) and Kumaresan et al. (2006).

\section{References}

Badade, D.S., Warade, S.D. and Gaikwad, S.K. (2001). Genetic divergence in bottle gourd. J. of Maharastra Agri. Uni.26:2, 137-139.

Gupta, V, Kishore, N and Pratap, P.S. (2006). Diallel analyses for combining ability in bitter gourd. Crop Res. (Hissar) 32 (3) : 406-410.

Islam, M.T. (2004). Bulletin of the Institute of Tropical Agriculture, Kyushu University. 27:19-24.

Kalloo, G. (1993). Loofah-Luff S.P.P. (ed.). 
Genetic Improvement of Vegetable Crops. pp. 265-266.

Kumaresan, G.R., Makesh, S. and Ramaswamy, N. (2006a). Studies on heterosis in snake gourd (Trichosanthes anguina L.). Crop Res. (Hissar). 31: 1, 99-102.

Maurya, P. K. (2010) Heterosis and combining ability analysis in bottle gourd (Lagenaria siceraria (Molina.) standl.). M. Sc. Thesis, Submitted to N. D. Univ. of agric. \& Tech., Kumarganj, Faizabad.

Singh, N.P., Dubey, A.K. and Srivastava, J.P.
(2006). Genetic architecture of yield and its component traits in bottle gourd (Lagenaria siceraria (Mol.) Standl.). Veg. Sci. 33 (2): 188-189.

Varalakshmi, B. Reddy, Y. N. Reddy, B. M. (1994). Genetic divergence in ridge gourd (Luffa acutangula (Roxb.) L.). Journal of Genetics \& Breeding; 1994. 48(2):131-134.

Virendra K R, D.; Singh, T. B. and Rajwade, V. B. (2010). Combining ability studies of quantitative traits in sponge gourd [Luffa cylindrica (Roem) L.]. New Agriculturist, 21(1/2):13-18. 10.

\section{How to cite this article:}

Rajneesh and Singh, V.B. 2018. General and Specific Combining Ability Analysis for Growth and Yield Attributes in Sponge Gourd [Luffa Cylindrica (Roem) L.] Int.J.Curr.Microbiol.App.Sci. 7(12): 3567-3577. doi: https://doi.org/10.20546/ijcmas.2018.712.404 